\title{
Televised medical talk shows-what they recommend and the evidence to support their recommendations: a prospective observational study
}

\author{
(c) $(1)$ (8)
}

Christina Korownyk associate professor of family medicine ${ }^{1}$, Michael R Kolber associate professor of family medicine ${ }^{1}$, James McCormack professor of pharmacy ${ }^{3}$, Vanessa Lam research assistant ${ }^{2}$, Kate Overbo research assistant ${ }^{2}$, Candra Cotton pharmacist ${ }^{1}$, Caitlin Finley research assistant ${ }^{1}$, Ricky D Turgeon pharmacist ${ }^{3}$, Scott Garrison associate professor of family medicine ${ }^{1}$, Adrienne $\mathrm{J}$ Lindblad associate clinical professor of family medicine ${ }^{1}$, Hoan Linh Banh associate professor of family medicine ${ }^{1}$, Denise Campbell-Scherer associate professor of family medicine ${ }^{1}$, Ben Vandermeer biostatistician $^{4}, \mathrm{G}$ Michael Allan professor of family medicine ${ }^{1}$

${ }^{1}$ Department of Family Medicine, University of Alberta, 6-10 University Terrace, Edmonton, Alberta, Canada T6G $2 \mathrm{~T} 4 ;{ }^{2} \mathrm{Faculty}$ of Medicine and Dentistry, University of Alberta, 2J2.00 WC Mackenzie Health Sciences Centre, Edmonton, Alberta, Canada T6G 2R7; ${ }^{3}$ Faculty of Pharmaceutical Sciences, University of British Columbia, 2405 Wesbrook Mall, Vancouver, BC, Canada V6T 1Z3; ${ }^{4}$ Alberta Research Centre for Health Evidence, University of Alberta, Edmonton Clinic Health Academy, 11405-87 Avenue, Edmonton, Alberta, Canada T6G 1C9

\begin{abstract}
Objective To determine the quality of health recommendations and claims made on popular medical talk shows.

Design Prospective observational study.

Setting Mainstream television media.

Sources Internationally syndicated medical television talk shows that air daily (The $\mathrm{DrOz}$ Show and The Doctors).

Interventions Investigators randomly selected 40 episodes of each of The $\mathrm{DrOz}$ Show and The Doctors from early 2013 and identified and evaluated all recommendations made on each program. A group of experienced evidence reviewers independently searched for, and evaluated as a team, evidence to support 80 randomly selected recommendations from each show.
\end{abstract}

Main outcomes measures Percentage of recommendations that are supported by evidence as determined by a team of experienced evidence reviewers. Secondary outcomes included topics discussed, the number of recommendations made on the shows, and the types and details of recommendations that were made.

Results We could find at least a case study or better evidence to support $54 \%$ ( $95 \%$ confidence interval $47 \%$ to $62 \%$ ) of the 160 recommendations
(80 from each show). For recommendations in The $\mathrm{DrOz}$ Show, evidence supported $46 \%$, contradicted $15 \%$, and was not found for $39 \%$. For recommendations in The Doctors, evidence supported 63\%, contradicted $14 \%$, and was not found for $24 \%$. Believable or somewhat believable evidence supported $33 \%$ of the recommendations on $\mathrm{The} \mathrm{DrOz}$ Show and 53\% on The Doctors. On average, The $\mathrm{Dr} \mathrm{Oz}$ Show had 12 recommendations per episode and The Doctors 11 . The most common recommendation category on $\mathrm{The} \mathrm{DrOz}$ Show was dietary advice (39\%) and on The Doctors was to consult a healthcare provider (18\%). A specific benefit was described for $43 \%$ and $41 \%$ of the recommendations made on the shows respectively. The magnitude of benefit was described for $17 \%$ of the recommendations on The $\mathrm{Dr} \mathrm{Oz}$ Show and $11 \%$ on The Doctors. Disclosure of potential conflicts of interest accompanied $0.4 \%$ of recommendations.

Conclusions Recommendations made on medical talk shows often lack adequate information on specific benefits or the magnitude of the effects of these benefits. Approximately half of the recommendations have either no evidence or are contradicted by the best available evidence. Potential conflicts of interest are rarely addressed. The public should be skeptical about recommendations made on medical talk shows.

Additional details of methods used and changes made to study protocol 


\section{Introduction}

Mass media in the form of television, radio and printed material are frequently used to deliver medical information to the public. Research suggests that mass media can improve public knowledge $^{1}$ and potentially improve health behaviors. ${ }^{2}$ Television is one of the most important mass media sources of health information. ${ }^{34}$ However, concerns have been raised about the quality, completeness and accuracy of medical information covered in the news media, ${ }^{5-8}$ and television news media is no exception. ${ }^{78}$ The quality of information outside of the news media has not been examined.

According to Nielsen's report, American citizens spend an average of over five hours a day watching television. ${ }^{9}$

International health information programs, such as $\mathrm{The} \mathrm{Dr} \mathrm{Oz}$ Show and The Doctors have become a regular part of television broadcasting. In the 2012-13 season, The Dr Oz Show was consistently ranked in the top five talk shows in America with an average of 2.9 million viewers per day, while The Doctors had a high of 2.3 million viewers. ${ }^{10}{ }^{11}$ In the 2012 Greatist report, Dr Mehmet $\mathrm{Oz}$ and Dr Travis Stork (one of the hosts of The Doctors) were both included in the top 100 health and fitness influencers. ${ }^{12}$

Popular television talk shows such as The $\mathrm{Dr} O z$ Show often engender skepticism and criticism from medical professionals. ${ }^{13-15}$ However, no research has systematically examined the content of the medical information provided on these talk shows. Our objective was to review the most popular medical talk shows on television, to (1) determine the type of recommendations and claims given and the details provided, and (2) search for and evaluate the evidence behind these recommendations.

\section{Methods \\ Programs}

We searched for internationally syndicated medical or health television talk shows that aired daily (weekdays). Two internationally syndicated medical or health television talk shows (The Dr Oz Show and The Doctors) were identified by internet search. We prospectively recorded all episodes of The Doctors from 11 January to 1 May 2013 (79 episodes) and The $\mathrm{Dr} O z$ Show from 7 January to 1 May 2013 (78 episodes).

\section{Data collection and outcome measures}

Three members of the research group (GMA, MRK, CK) reviewed two episodes of each show from the previous year (2012) to help inform the development of a data collection spreadsheet.

The primary outcome was the percentage of recommendations and claims (henceforth referred to as recommendations) supported by evidence as determined by a team of experienced evidence reviewers. Secondary outcomes included topics discussed, the number of recommendations made, the types and details of the recommendations, and general episode characteristics.

We had no previous information on which to base an estimation of the needed sample size to make a reasonable estimate of the number of recommendations that were evidence based. Based on direct observation of two of each of the shows, we hypothesized that $50 \%$ of the recommendations would be supported by evidence, then calculated that 158 recommendations would give an $80 \%$ chance that the $95 \%$ confidence interval would have a precision within $\pm 10 \%$. We estimated there would be at least two definitive or stronger recommendations per episode. Therefore, to attain 158 recommendations, we needed to review 80 episodes. Using a random number generator, 40 of the recorded episodes for each of The $\mathrm{Dr} \mathrm{Oz}$. Show and The Doctors were randomly selected for analysis.

Being unfamiliar with broadcast health information, we were unaware of the non-specific nature of many statements and recommendations given on medical television talk shows. In addition, the content of the medical information provided on talk shows has not previously been assessed systematically. This required us to use an iterative approach to the study design, ultimately resulting in three small changes to the protocol and one larger change (see data supplement for full details). A flow chart of the final study is presented in figure $1 \Downarrow$.

\section{Classification and content of recommendations}

Two reviewers (VL, KO) independently watched each randomly selected episode to document the topics discussed and the specific details surrounding recommendations. Further information on data extraction is included in the data supplement. To focus on stronger or clearer recommendations for the evidence review portion of the analysis, the reviewers attempted to delineate the more definitive recommendations. This was based on both the strength of the wording (for example, "Get your kids vaccinated" was considered a strong recommendation, whereas "Prescription retin-A helps with ear and other types of acne" was not) and the context in which the recommendation was made (for example, a recommendation may have been classified as strong if it was repeated multiple times). After independent review, data extraction was compared, disagreement of coding and classification was resolved by consensus or third party adjudication (CK, MRK, or GMA), and overall agreement was calculated. We found some of the initial classification of topics and recommendations included categories that were too broad. Therefore, two investigators $(\mathrm{CK}, \mathrm{JMcC})$ reclassified all topics and recommendations, again with a third investigator (GMA) resolving any uncertainties.

After preliminary review of the data, it was determined that more specific detail was needed to better quantify the type of information provided for all recommendations. Two new reviewers $(\mathrm{CC}, \mathrm{CF})$ independently watched the same randomly selected episodes and focused on the recommendations to identify if a benefit was described (such as "heart healthy"), if the benefit was specific (such as "reduces heart attacks"), if a magnitude of benefit was given (such as "reduced 5\%"), if costs were mentioned (such as "this supplement costs $\$ 5$ a month"), and if potential risks or harms were mentioned. In addition, the observers recorded any mention of potential conflict of interest with each recommendation or acknowledgement of potential conflicts of interest at any point during the show. After independent review, data extraction was compared, disagreement was resolved by consensus or third party adjudication (CK, MRK, or GMA), and overall agreement was calculated.

\section{Development of searchable questions and the evidence behind the recommendations}

We randomly selected 80 of the stronger recommendations from each show and developed a searchable question for each. Two healthcare providers from the group with medical literature searching skills (GMA, MRK, CK, JMcC, SG, AJL, HLB, DCS, RDT) independently searched for evidence supporting each recommendation. The searchable resources included the 


\section{Format of a typical medical talk show}

Shows are typically divided into 3-4 topics (range about 1-5), each topic runs about 12-15 minutes, often divided by commercial breaks

Each topic may have 4-5 recommendations within it (range about 0-10)

Recommendations may come from the host, guest, or rarely audience members

databases Pubmed/Medline, Embase, Cochrane Database of Systematic Reviews, Natural Standard Database, and Google. Maximum search time allotted was 1 hour per recommendation. If reviewers found high level evidence (a systematic review of randomized controlled trials or large randomized controlled trials) addressing the question or recommendation, the search was concluded. Original research that addressed patient oriented outcomes (such as mortality or quality of life) was assigned higher priority over surrogate markers or in vitro data. If several papers were available, reviewers selected the highest level evidence pertaining to the target audience of the shows (such as middle age adults versus pediatrics, and North American data). Reviewers recorded citations of all papers that were considered relevant and were encouraged to make notes regarding their search and evaluation of the evidence.

Originally each reviewer was also going to independently judge if the evidence was of sufficient consistency and believability to support the recommendations. However, challenges occurred with the non-specific nature of the recommendations and wide diversity of reviewer interpretation of the evidence (see data supplement). Therefore, the protocol was modified and four investigators (CK, MRK, JMcC, GMA) reviewed the evidence for each recommendation as a team. The investigators reviewed the recommendation and determined the ideal study design to answer the question but considered sources of evidence ranging from case reports to systematic reviews. While expert opinion is considered a level of evidence, these were excluded because all recommendations in the programs were, by definition, being made by experts. In cases where the answer remained unclear, an additional search was performed until the group was satisfied that a reasonable answer was found or that no evidence was readily available.

The evidence was evaluated for consistency and believability. Consistency was defined as both internal (within the study) and external (between studies). Believability was based on the quality, quantity, and type of evidence available. The group discussed each recommendation and voted on how believable (believable, somewhat believable, or not believable) the evidence was. When reviewing evidence, investigators used a relatively broad definition of support in an attempt to be as fair as possible (for example, see data supplement). The group also recorded how frequently "consult a healthcare provider" was mentioned in the 160 randomly selected stronger recommendations. All data are presented descriptively without statistical comparison. The $95 \%$ confidence intervals for the primary outcome were computed using the normal scores method.

\section{Results \\ Program topics}

Topic categorizations from the 80 randomly selected episodes (40 of The Dr Oz Show and 40 of The Doctors) are shown in figure $2 \Downarrow$. The most common show topic discussed on both The $\operatorname{Dr} \mathrm{Oz}$ Show and The Doctors was general medical advice $-32.0 \%$ (40/125) and $65.5 \%$ (74/113) respectively. The second most common topic on both shows, $24.8 \%$ (31/125) and $8.8 \%(10 / 113)$ respectively, was non-weight loss dietary advice (such as immune boosting diets). The combination of dietary advice and weight loss accounted for $43.2 \%(54 / 125)$ of all topics discussed on The $\mathrm{Dr} O z$ Show and 16.8\% (19/113) for The Doctors.

\section{Program recommendations}

We identified 479 recommendations for The Dr Oz Show (291 stronger recommendations) and 445 recommendations for The Doctors, (219 stronger recommendations). On average, each episode of The Dr Oz Show had 12 recommendations, while each episode of The Doctors had 11 recommendations.

\section{Recommendation categories}

The categories of the recommendations for both shows are shown in figure $3 \Downarrow$. The most common recommendation in The $\operatorname{DrO}$. Show (39.2\%, 188/479) was dietary advice (such as "Carb load your plate at breakfast"), while in The Doctors (17.8\%, $79 / 445$ ) it was to consult a healthcare provider (such as "Go to your primary care doctor or talk to their nurse before going to the ER to help relieve the load in the ER"). For the 160 recommendations that were randomized for evidence review, The $\mathrm{Dr} O \mathrm{O}$. Show recommended consulting a healthcare professional $9 \%(7 / 80)$ of the time compared with $33 \%$ (26/80) of the time on The Doctors.

\section{Recommendation details}

The details of benefits, harms, and costs around the recommendations are shown in table $1 \Downarrow$. The benefit of the recommendation was not specific $57.4-58.7 \%$ of the time. For example, a recommendation from $\mathrm{The} \mathrm{Dr} \mathrm{Oz}$ Show that vitamin E improves brainpower would be considered a benefit but not considered specific or measurable, and the magnitude of the increase in brainpower was not discussed. Magnitude of the potential benefit was mentioned in $11.0-16.5 \%$ of the recommendations, often in relative rather than absolute terms (for example, vitamin $\mathrm{E}$ in foods cuts risk of Alzheimer's disease by $25-70 \%$ ). Recommendations were made by the host(s) $26 \%$ (125/479) of the time on The Dr Oz Show and 65\% (290/445) of the time on The Doctors. Guests made 65\% (310/479) of the recommendations on The $\mathrm{Dr} O z$. Show and 33\% (146/445) on The Doctors.

Both the host(s) and guests made the recommendations 9\% (44/479) of the time on The Dr Oz Show and 2\% (7/445) of the time on The Doctors. Acknowledgment of a potential conflict of interest was identified four times over the 924 recommendations.

\section{Evidence base for the stronger recommendations}

Evidence assessment for 160 randomly selected recommendations is presented in table $2 \Downarrow$. Overall, we found that 87 of the 160 recommendations $(54 \%, 95 \%$ confidence interval $47 \%$ to $62 \%$ ) had some level of published evidence to support them. Believable or somewhat believable evidence supported 33\% of the recommendations on The $\mathrm{Dr} \mathrm{Oz}$ Show and $53 \%$ on The Doctors. We found believable or somewhat believable evidence against $11 \%$ and $13 \%$ of the 
recommendations on the The Dr Oz Show and The Doctors, respectively.

Clinical outcomes (versus surrogate) were used for $85 \%$ of The Dr Oz Show and $98 \%$ of The Doctors recommendations. At times these recommendations were justified via an intermediate or surrogate outcome that in turn would result in clinical outcome. For example, flossing every day decreases inflammation of the gums, and this inflammation causes aging.

\section{Discussion \\ Principal findings}

In our review of the two most popular medical talk shows, we found that general medical advice was the most common topic addressed on both shows. This broad category includes advice that could not otherwise be categorized into dietary, weight loss, exercise, cosmetic or counseling. The most common recommendations differed between the shows. On The $\mathrm{Dr} O z$ Show the most common recommendations were dietary advice, while the most common recommendation on The Doctors was to consult a healthcare professional. Dietary recommendations were almost four times more common (39\% v 10\%) on The Dr $O z$ Show than on The Doctors. Dietary recommendations on The $\mathrm{Dr} O z$ Show were close to eight times more common (39\% $v 5 \%)$ than exercise recommendations. Similarly, dietary recommendations were twice as common as exercise recommendations $(10 \% v 5 \%)$ on The Doctors.

For both shows, a specific benefit was mentioned for only about $40 \%$ of the recommendations. The magnitude of benefit $(<20 \%)$, potential harms $(<10 \%)$, and costs $(<15 \%)$ were less commonly mentioned. Thus, anyone who followed the advice provided would be doing so on the basis of a trust in the host or guest rather than through a balanced explanation of benefits, harms, and costs. The near absence of potential conflict of interest reporting $(<1 \%)$ further challenges viewers' ability to balance the information provided.

Roughly a third of the recommendations on The Dr Oz Show and half of the recommendations on The Doctors were based on believable or somewhat believable evidence. Evidence was believable or somewhat believable against a recommendation for 1 in 8-10 recommendations. For slightly over 1 in 3 and 1 in 4 of the recommendations for The $\mathrm{Dr} O z$. Show and The Doctors respectively, no evidence could be found. This is despite us being quite liberal in the type and amount of evidence we required. The percentage of medical practice in the real world that is evidence based is difficult to ascertain, although one review reported an average of $78 \%$ of medical interventions were based on some form of "compelling" evidence. ${ }^{16}$ Comparisons are difficult, however, because the types of evidence that was required to determine this is considerably varied.

\section{Strengths and limitations of study}

Limitations of this study include the inherent complexity of the shows, including the subjective nature of the recommendations such as distinguishing between what was said and what was implied. To our knowledge, a detailed analysis like this has not been attempted before, and thus determining the best approach to gathering and quantifying the information was iterative.

Although the protocol was planned ahead, it was adapted due to unanticipated challenges in data collection and interpretation. While this was a limitation, it also represents a lack of pre-disposition bias.
Developing searchable questions for each recommendation was difficult because less than $50 \%$ of recommendations were associated with a specific benefit. Again, we were liberal with our question development and search for evidence. Our original approach of having two independent experienced evidence reviewers (out of a pool of nine) review and categorize the evidence for each recommendation led to too much inconsistency due to the variability of the recommendations and the type of evidence required for each recommendation. Our adapted approach of four reviewers examining and discussing the evidence for recommendations was also imperfect, but we believe this method was the best way to provide a consistent review of the evidence for these highly subjective recommendations. During evidence review, some of the recommendations may have been standard or commonly recommended medical care or public health advice (such as "sneezing into your elbow prevents the spread of germs"), but we could not find evidence to support the recommendation. Although these were uncommon, it increased somewhat the proportion of recommendations with no evidence.

Finally, the time period selected for recording may not be reflective of the shows over multiple years, although it is unlikely that sampling episodes over a different time would change the results meaningfully. This is the first study to assess recommendations made on popular medical television talk shows from an evidence based perspective, and the strengths of the study include dual review at all levels, detailed data extraction, committee discussion for evidence assessment, and an iterative methodology.

\section{Conclusions}

Consumers should be skeptical about any recommendations provided on television medical talk shows, as details are limited and only a third to one half of recommendations are based on believable or somewhat believable evidence. An interesting question is whether we should expect medical talk shows to provide more than entertainment. Future studies may be directed at determining what viewers hope to obtain from watching these shows, and if the airing of these shows results in behavior changes related to specific recommendations. If the shows are perceived as providing medical information or advice, viewers need to realize that the recommendations may not be supported by higher evidence or presented with enough balanced information to adequately inform decision making. Decisions around healthcare issues are often challenging and require much more than non-specific recommendations based on little or no evidence from media health professionals. Patients would do well to ask healthcare providers specific questions about the benefits and harms, along with the magnitude of the effect (in absolute numbers), and the costs and inconveniences of any recommendation.

Contributors: CK, GMA, MRK, JMcC conceived and designed the study. CK, GMA, MRK, JMcC, VL, KO, CC, CF, RDT, AJL, HLB, SG, DC-S, BV acquired, analysed, or interpreted data. CK, GMA, JMcC drafted the manuscript. CK, GMA, MRK, JMcC, VL, KO, CC, CF, RDT, AJL, HLB, SG, DC-S, BV critically revised the manuscript for important intellectual content. BV, GMA conducted statistical analysis. CK, GMA obtained funding. GMA provided administrative, technical, and material support. GMA, JMcC, MRK, CK supervised the study.

Funding: This study was supported in part by the David and Beatrice Reidford Research Scholarship through the University of Alberta (valued at $\$ 2400$ ). The sponsors had no role in the design and conduct of the study; collection, management, analysis, and interpretation of the data; 


\section{What is known on this topic}

Television is an important source of health information for the general public

Popular medical television talk shows frequently engender skepticism and criticism from medical professionals

No research has systematically examined the content of the medical information provided on these talk shows

\section{What this study adds}

Recommendations made on popular medical television talk shows often lack adequate information on specific benefits, magnitude of benefits, and harms, and do not facilitate informed decision making

Approximately half of the recommendations have either no evidence or are contradicted by the best available evidence. Potential conflicts of interest are rarely addressed

The public should be skeptical about recommendations made on medical talk shows

preparation, review, or approval of the manuscript; and decision to submit the manuscript for publication.

Competing interests: All authors have completed the ICMJE uniform disclosure form at www.icmje.org/coi_disclosure.pdf and declare: no support from any organisation for the submitted work; no financial relationships with any organisations that might have an interest in the submitted work in the previous three years; no other relationships or activities that could appear to have influenced the submitted work. Ethical approval: Not required.

Transparency: CK affirms that the manuscript is an honest, accurate, and transparent account of the study being reported; that no important aspects of the study have been omitted; and that any discrepancies from the study as planned have been explained.

Data sharing: For further information on the dataset, please contact the corresponding author at tina.korownyk@ualberta.ca

Compliance with reporting guidelines: As we are unaware of other similar studies, there is not a template with which to perform this type of study. We used STROBE guidelines where possible but could not adhere to it rigidly.

1 Marx JJ, Nedelmann M, Haertle B, Dieterich M, Eicke BM. An educational multimedia campaign has differential effects on public stroke knowledge and care-seeking behavior. J Neurol 2008;255:378-84

2 Bala MM, Strzeszynski L, Topor-Madry R, Cahill K. Mass media interventions for smoking cessation in adults. Cochrane Database Syst Rev 2013;6:CD004704.

3 Oakley A, Bendelow G, Barnes J, Buchanan M, Husain OA. Health and cancer prevention: knowledge and beliefs of children and young people. BMJ 1995;310:1029-33.
4 Chew F, Palmer S, Slonska Z, Subbiah K. Enhancing health knowledge, health beliefs, and health behavior in Poland through a health promoting television program series. $J$ Health Commun 2002; 7:179-96.

5 Cassels A, Hughes MA, Cole C, Mintzes B, Lexchin J, McCormack JP. Drugs in the news: an analysis of Canadian newspaper coverage of new prescription drugs. CMAJ 2003;168:1133-7.

6 Milazzo S, Ernst E. Newspaper coverage of complementary and alternative therapies for cancer-UK 2002-2004. Support Care Cancer 2006;14:885-9.

7 Tong A, Chapman S, Sainsbury P, Craig JC. An analysis of media coverage on the prevention and early detection of CKD in Australia. Am J Kidney Dis 2008;52:159-70.

8 Moynihan R, Bero L, Ross-Degnan D, Henry D, Lee K, Watkins J, et al. Coverage by the news media of the benefits and risks of medications. N Engl J Med 2000;342:1645-50.

9 Statistic Brain. Television watching statistics. 2012. www.statisticbrain.com/televisionwatching-statistics/.

10 The Hollywood Reporter. 'Dr. Oz' and 'Ellen' see ratings growth, 'Kelly \& Michael' topping 2011 in demo. 2012. www.hollywoodreporter.com/news/dr-oz-ellen-see-ratings-387407.

11 The Hollywood Reporter. Updated: TV ratings: 'Dr. Phil' tops talkers during February sweep, 'Dr. Oz' sees steep declines. 2013. www.hollywoodreporter.com/news/updatedtv-ratings-dr-phil-427733.

12 Greatist. 2012. The 100 most influential people in health and fitness. http://greatist.com/ health/most-influential-health-fitness-people.

13 Inoue-Choi M, Oppeneer SJ, Robien K. Reality check: there is no such thing as a miracle food. Nutr Cancer 2013;65:165-8.

14 Hagenbruch JF. Dr. Oz sells out the Hippocratic Oath. CDS Rev 2013;106:5.

15 Cifu AS. Why Dr. Oz makes us crazy. J Gen Intern Med 2014;29:417-8.

16 Imrie R, Ramey DW. The evidence for evidence-based medicine. Complement Ther Med 2000;8:123-6.

Accepted: 19 November 2014

\section{Cite this as: BMJ 2014;349:97346}

This is an Open Access article distributed in accordance with the Creative Commons Attribution Non Commercial (CC BY-NC 4.0) license, which permits others to distribute, remix, adapt, build upon this work non-commercially, and license their derivative works on different terms, provided the original work is properly cited and the use is non-commercial. See: http://creativecommons.org/licenses/by-nc/4.0/. 


\section{Tables}

Table 1| Details of benefits, harms, and costs associated with each recommendation made in samples of medical television talk shows The Dr Oz Show and The Doctors

\begin{tabular}{lcc} 
& \multicolumn{2}{c}{ No (\%) of recommendations } \\
\cline { 2 - 3 } Benefit of recommendation mentioned & $453(94.6)$ & $402(90.3)$ \\
\hline Benefit was specific & $204(42.6)$ & $184(41.3)$ \\
\hline Magnitude of benefit mentioned & $79(16.5)$ & $49(11.0)$ \\
\hline Possible harms mentioned & $47(9.8)$ & $34(7.6)$ \\
\hline Cost mentioned & $60(12.5)$ & $14(3.1)$ \\
\hline Potential conflict of interest declared or mentioned & 1 time & 3 times \\
\hline
\end{tabular}


Table 2| Evidence for 80 randomly selected recommendations from each of the medical television talk shows The Dr Oz Show and The Doctors

\begin{tabular}{|c|c|c|c|}
\hline & \multirow[b]{2}{*}{ Evidence believable } & \multicolumn{2}{|c|}{ Number $(\%)$ of recommendations $(n=80)$} \\
\hline & & By believability & Overall \\
\hline \multicolumn{4}{|l|}{ The Dr Oz Show } \\
\hline \multirow[t]{3}{*}{ Evidence agrees } & Yes & $17(21.3)$ & $47(46.3)$ \\
\hline & Intermediate & $9(11.3)$ & \\
\hline & No & $11(13.8)$ & \\
\hline \multirow[t]{3}{*}{ Evidence disagrees } & Yes & $4(5.0)$ & $12(15.0)$ \\
\hline & Intermediate & $5(6.3)$ & \\
\hline & No & $3(3.8)$ & \\
\hline No evidence & - & - & $31(38.8)$ \\
\hline \multicolumn{4}{|l|}{ The Doctors } \\
\hline \multirow[t]{3}{*}{ Evidence agrees } & Yes & $26(32.5)$ & $50(62.5)$ \\
\hline & Intermediate & $16(20.0)$ & \\
\hline & No & $8(10.0)$ & \\
\hline \multirow[t]{3}{*}{ Evidence disagrees } & Yes & $3(3.8)$ & $11(13.8)$ \\
\hline & Intermediate & $7(8.8)$ & \\
\hline & No & $1(1.3)$ & \\
\hline No evidence & - & - & $19(23.8)$ \\
\hline
\end{tabular}




\section{Figures}

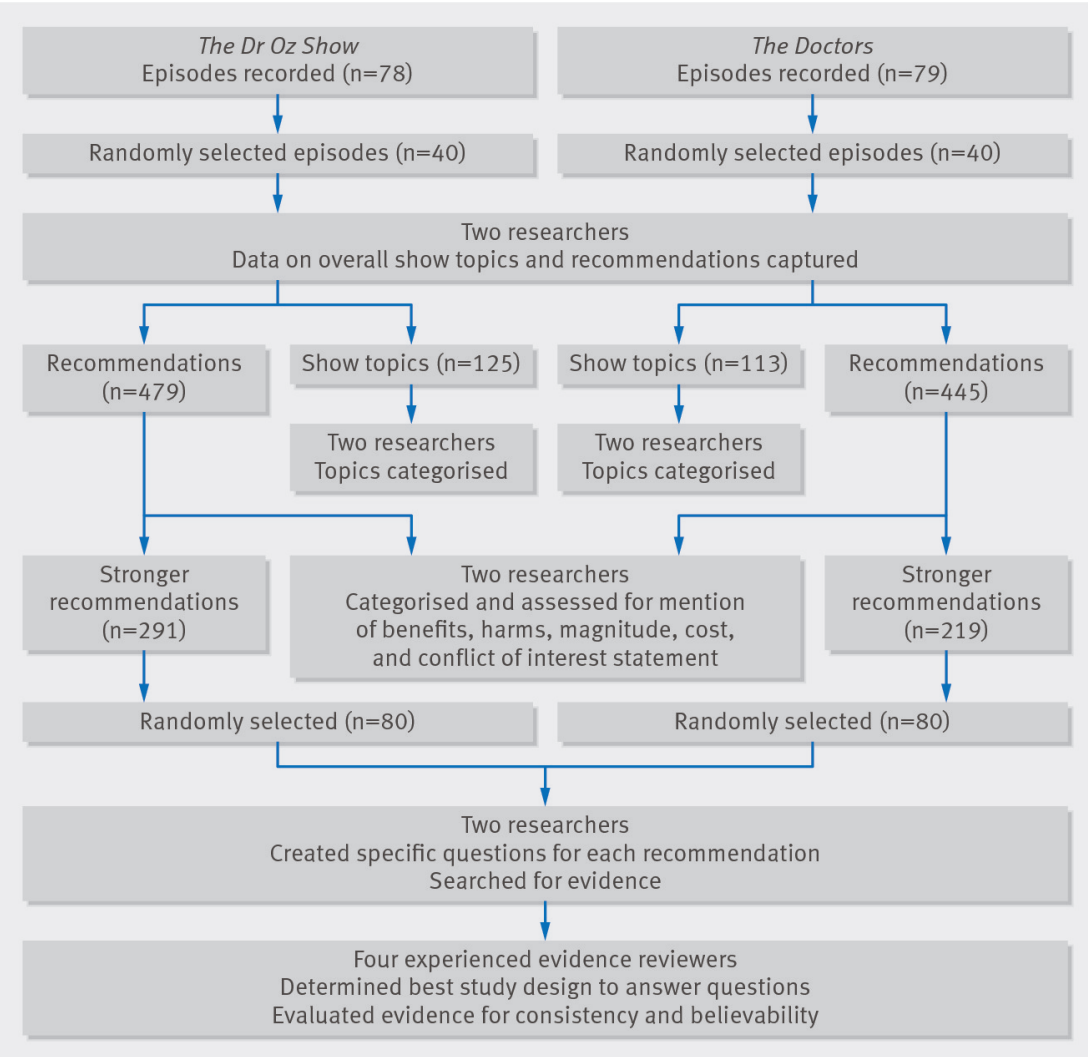

Fig 1 Flow chart of study design

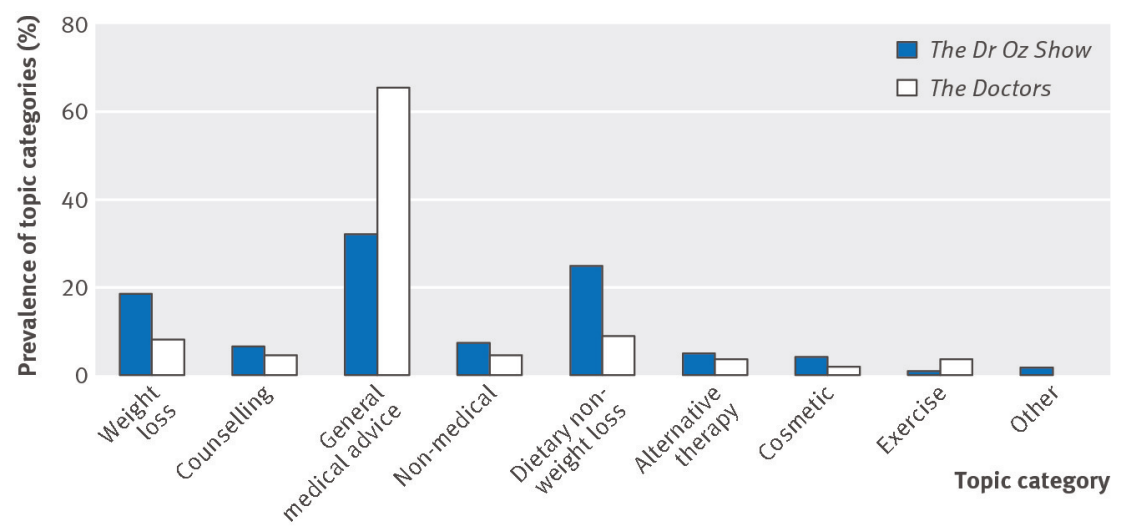

General medical advice ranges from advice around tonsillitis and influenza to winter health tips from summer Olympians Dietary non-weight loss refers to diets to improve health (example, boost immune system)

Fig 2 Prevalence of topic categories in 40 episodes of each of The Dr Oz Show (125 topics) and The Doctors (113 topics) 


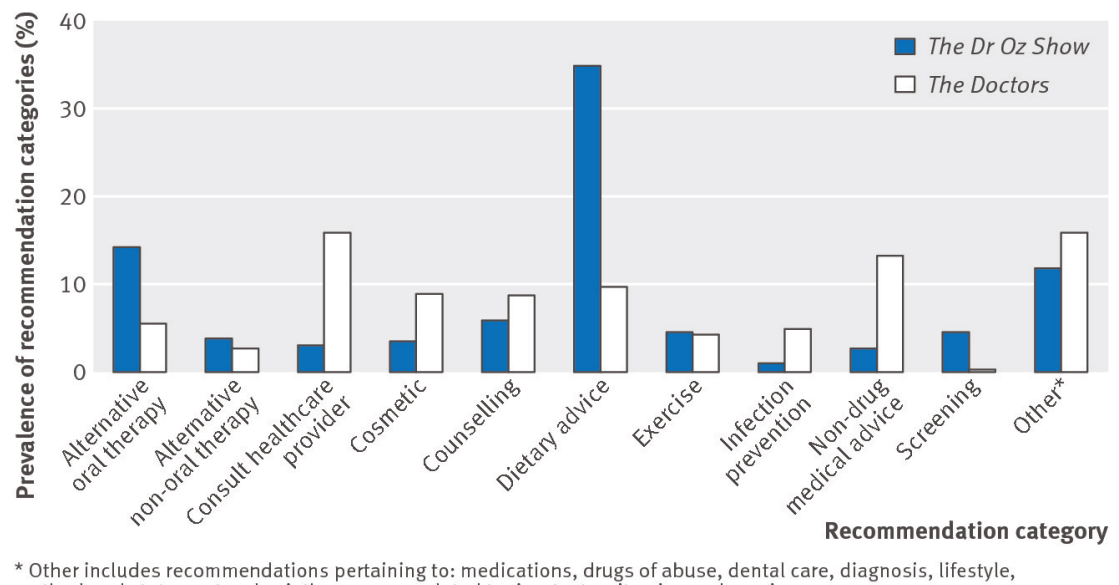

* Other includes recommendations pertaining to: medications, drugs of abuse, dental ca
motherhood statements, physiotherapy, sex related topics, tests, vitamins and vaccines

Fig 3 Prevalence of recommendation categories in 40 episodes of each of The $\mathrm{DrOz}$ Show (479 recommendations) and The Doctors (445 recommendations). 